\title{
Auctioning Game based Demand Response Scheduling in Smart Grid
}

\author{
Ding Li, Sudharman K. Jayaweera and Asal Naseri \\ Department of Electrical and Computer Engineering, University of New Mexico, Albuquerque, USA \\ Email: \{liding, jayaweera, anaseri2\}@ece.unm.edu
}

\begin{abstract}
Matching demand to supply is one of the key features of smart grid infrastructure. Transforming conventional static customers into active participants who interact with the electrical utility in real time is the central idea of Demand Response (DR) $\backslash$ Demand Side Management (DSM) in smart grid. In this paper, we decouple utility cost minimization and customer social welfare maximization into two stages. Since the utility is usually more risk averse than risk neutral in real life, this decoupling approach is more realistic than the usually adopted optimization setup, in which the two objectives are combined in a single weighted sum. With a block processing model introduced, in the first stage a convex optimization problem is formulated to minimize utility's generation cost and delay operation cost. An optimal load demand scheduling solution, of the form of waterfilling, is derived analytically. Based on the optimal load profile generated in this first stage, repeated Vickrey auctions over time intervals are adopted to allocate load demands among customers while maximizing the social welfare. Despite the fact that truthful bidding is a weakly dominant strategy for all customers in the auctioning game, collusive equilibria do exist and jeopardize utility's profit severely. Analysis on the structure of the Bayesian Nash equilibrium solutions shows that by introducing a positive reserve price the Vickrey auction can be made to be more robust against such collusion by customers. Moreover the corresponding Bayesian Nash equilibrium is essentially unique and guarantees the basic profit of the utility. We further discuss how customers' valuations and bidding strategies change over time for the repeated Vickrey auction model. Simulation results emphasizing the influences of reserve price and time interval size on utility's profit is also presented.
\end{abstract}

Index Terms-Demand response, repeated Vickrey auctions, Bayesian Nash equilibria, block scheduling.

\section{INTRODUCTION}

In a conventional electrical power market, power generation is usually required to match to power demand. This is because the distributed nature of power demands, as well as the different energy consumption behaviors of customers in the power network, make power demand fluctuating over time and difficult to be controlled precisely. This behavior is expected to become more significant as high penetration of renewable generations and PHEVs appear in generation side and consumption side separately. As a result of the highly time varying generation and consumption profiles, the utility needs to provide enough electrical power to meet peak demand rather than the average to prevent potential blackout events. However, this static and centralized generation pattern is apparently inefficient and thus costly. For example, the U.S. national load factor is about $55 \%$, and $10 \%$ of generation and $25 \%$ of distribution facilities are used less than 400 hours per year, i.e., $5 \%$ of the time [1]. Finding possible approaches to improve this inefficient performance is one of the strong incentives to consider a smart grid. In smart grid infrastructure, the key feature of matching demand to supply by transforming currently static consumers into active participants is the central idea of Demand Response (DR) and Demand Side Management (DSM) [2] which can greatly improve power system efficiency and thus yield huge savings.

The literature on DR $\backslash$ DSM so far fall into three main categories. The first category of approaches adopt optimization techniques [3]-[5]. The optimization objectives are usually minimizing utility's cost, maximizing social welfare or a weighted combination of them. It turns out that these optimization problems are usually difficult to solve analytically. For example, under the constraint of utility's generation capacity, the load demands of different customers are coupled with each other making the problem intractable. Thus the solutions in this category are usually algorithm based and might only guarantee suboptimal results. The second category of approaches adopt ideas similar to random access protocols in data communications [6], [7]. These approaches usually assume that customers in the power network are completely "blind" with respect to each other and can only communicate with the utility: Customers send load demands to the utility and compete for the limited power generated over time. The utility deals with these demands according to carefully selected protocols by supporting, postponing or declining them. However, the lack of knowledge of future power demands makes these approaches inefficient on demand scheduling over time. Finally, the third category contains game theoretic approaches. In [8], a network traffic model was adopted and the equivalence between congestion games and potential games was utilized to analyze the equilibrium solutions. In [9], both scenarios under which customers are price taking in a competing market and price anticipating in an oligopolistic market were discussed.

The main contribution of this paper is that we propose a decoupling approach which divides the two objectives: utility cost minimization and customers social welfare maximization into two stages. In the first stage, on receiving the initially submitted load demands from customers, the utility generates an optimal load profile over time that minimizes its cost under the generation capacity constraint. In the second stage, based on the load demand profile obtained in the previous stage, repeated auctions are adopted to allocate loads among 
customers in the network to maximize the social welfare. This decoupling gives better description of electricity networks than the usually adopted optimization scheme does, in which the two objectives are combined into a weighted sum. Since in reality the utility always tries to avoid blackout and brownout events, it is more risk averse than risk neutral.

The rest of the paper is organized as follows: In section II we formulate the problem with a block processing model. In order to minimize the utility's cost, a convex optimization problem is formulated and solved analytically in section III. In section IV, the repeated Vickrey auction model is adopted as the distributed scheduling approach to allocate load demands among customers while maximizing the social welfare. Detailed analysis on the structure of the Bayesian Nash equilibrium solution set, as well as several desired properties of the auctioning game are discussed in section V. The conclusions from this study are given in Section VI.

\section{PROBLEM Formation}

We assume an electricity market consisting of one electrical utility and $K$ customers. A block processing model is adopted here in which load demands are scheduled in a periodic blockby-block manner. Each block consists of $I$ time intervals and the size of each time interval is $T$ hours. The utility and customers interact as follows: At the beginning of each time block, all customers submit their load demands of the current time block to the utility based on their energy requirements and electricity pricing information. The load demands from customers consist of two parts: nonflexible load demands and flexible load demands. The nonflexible load demands reflect the basic energy requirements of customers, which specify how much electrical energy is needed during each time interval of a time block. We denote by $l_{i, k}^{N}$ and $\tilde{l}_{i, k}^{F}(i=1,2, \ldots, I$, $k=1,2, \ldots, K)$ the nonflexible and flexible load demands from customer $k$ in time interval $i$, denote by $l_{i}^{N}=\sum_{k=1}^{K} l_{i, k}^{N}$ and $\tilde{l}_{i}^{F}=\sum_{k=1}^{K} \tilde{l}_{i, k}^{F}$ the total nonflexible and flexible load demands over all customers in time interval $i$, similarly $l_{N}=$ $\sum_{i=1}^{I} l_{i}^{N}$ and $\tilde{l}_{F}=\sum_{i=1}^{I} \tilde{l}_{i}^{F}$ denote the total nonflexible and flexible load demands during that time block. We assume that the utility guarantees supporting all nonflexible load demands during each specified time interval. Thus, in any time interval the sum of nonflexible load demands over all customers is assumed to be no greater than the generation capacity of the utility. On the other hand we assume that no customer cheats on its nonflexible load demand, e.g. declaring more nonflexible load demand than its actual basic requirement. This can be achieved by having a regulatory authority in a real electricity market. We assume that despite the time interval information specified by flexible load demands, from the beginning of a time block all customers want their flexible load demands be supported as early as possible. Energy support from the utility during later time intervals induces a delay cost which is an increasing function of both the delay time and the amount of energy that has been delayed, due to the dissatisfaction of customers.
Upon receiving customers' load demands, the utility checks the generation capacity constraint and determines an optimal generation profile that minimizes its cost over time. The amount of flexible load demand after load reallocation in time interval $i$ is denoted by $x_{i}$ and we assume that the utility provides constant power of $\frac{l_{i}^{N}+x_{i}}{T}$ within each time interval. Based on the optimal load profile $x_{i}(i=1,2, \ldots, I)$, the electrical energy is further allocated among customers in a way that maximizes the social welfare. The whole interaction procedure between the utility and customers is illustrated in figure 1 .

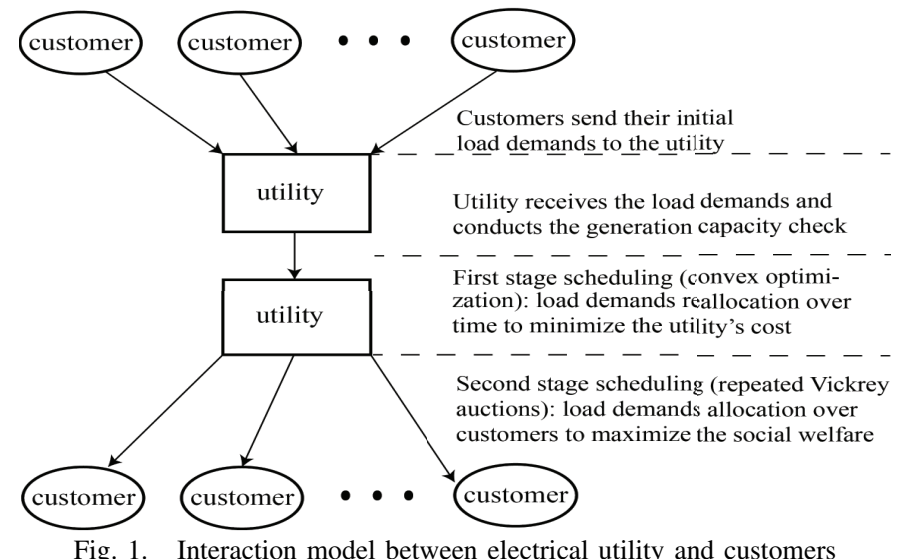

Fig. 1. Interaction model between electrical utility and customers III. UTILITY OPTIMIZATION: LOAD DEMAND SCHEDULING OVER TIME

In the first stage, we formulate and solve the problem of minimizing the utility cost by scheduling load demands over time intervals, assuming specific generation cost and delay cost forms. We assume that in time interval $i$, the generation cost per unit energy (in monetary measure) is a linear function of the total load demands in that time interval, say, $\alpha\left(l_{i}^{N}+x_{i}\right)$, where $\alpha$ is a positive scaling factor [10]. Thus the generation cost for time interval $i$ and for the entire time block are given by $C_{g, i}=\alpha\left(l_{i}^{N}+x_{i}\right)^{2}$ and $C_{g}=\sum_{i=1}^{I} C_{g, i}$ respectively. Moreover, we assume that if $x_{i}$ amount of load demands have been delayed by $i$ time intervals, the associated delay cost is given by $C_{d, i}=i T x_{i} \gamma^{-(I-i) T}$, where $\gamma$ is the positive delay cost scaling factor. Thus the total delay cost for a processing block is $C_{d}=\sum_{i=1}^{I} C_{d, i}$. Note that, when the total load demands (the nonflexible plus the flexible) in a time block is greater than the total energy that can be generated during that time block, no optimal reallocation solution exists unless the utility cuts down the flexible load demands. For fairness, the following strategy is adopted: If the total amount of load demand is greater than the total generation capability, the utility will cut every customer's flexible load demand by the same proportion to keep the total load demand equal to the generation capability, i.e, if $\tilde{l}_{F}+l_{N}>I L_{M}$, where $L_{M}$ is the constant generation capacity of utility in each time interval. The new flexible load demand is $l_{F}=\sum_{k=1}^{K} l_{k}^{F}$ and $l_{k}^{F}=\tilde{l}_{k}^{F}-\beta\left(\tilde{l}_{k}^{F}+l_{k}^{N}\right)$ where $\beta=\frac{\tilde{l}_{F}+l_{N}-I L_{M}}{\tilde{l}_{F}+l_{N}}$ for all $k$. Based on this centralized scheme, we may, without loss of generality, assume that the load demand in any time interval never exceed 
the generation capacity and thus drop the generation capacity constraint. Denoting by weighted sum $C=C_{g}+\delta C_{d}$ the total cost of utility, where $\delta$ is the weight coefficient for delay cost, we have the following optimization problem

$$
\begin{array}{ll}
\underset{\mathbf{x}}{\operatorname{minimize}} & C(\mathbf{x})=\alpha \sum_{i=1}^{I}\left(x_{i}+l_{i}^{N}\right)^{2}+\delta \sum_{i=1}^{I}\left(i T x_{i} \gamma^{-(I-i) T}\right) \\
\text { subject to } & -x_{i} \leq 0, \quad i=1,2, \ldots, I, \\
& \sum_{i=1}^{I} x_{i}-l_{F}=0 .
\end{array}
$$

Note that this optimization problem is convex, by solving the Karush-Kuhn-Tucker (KKT) conditions, the optimal solution can be written as

$$
x_{i}^{*}=\left\{\begin{array}{rl}
0 & \text { if } w^{*}<\hat{w}_{i} \\
w^{*}-\hat{w}_{i} & \text { if } w^{*} \geq \hat{w}_{i}
\end{array},\right.
$$

where $\hat{w}_{i}=l_{i}^{N}+\frac{\delta}{2 \alpha}\left(i T \gamma^{-(I-i) T}\right)$ and $w^{*}$ is the unique solution to $\sum_{i=1}^{I} \max \left(0, w^{*}-\hat{w}_{i}\right)=l_{F}$. For $\delta=0$ (i.e., delay cost is completely ignored), the solution (1) reduces to

$$
x_{i}^{*}=\left\{\begin{array}{rl}
0 & \text { if } w^{*}<l_{i}^{N} \\
w^{*}-l_{i}^{N} & \text { if } w^{*} \geq l_{i}^{N},
\end{array},\right.
$$

which is the so-called water-filling solution with water level $w^{*}$ [10]. To better interpret the solutions above, we consider an electricity network during a time block of $I=24$ intervals with $T=1$ hour. The customers' load demands of different time intervals are generated according to different distributions corresponding to time dependent electrical energy consumption behavior. Given a set of initial load demands, the optimal allocation results for different $\delta$ values are shown in figure 2 . It is seen that the solution (1) is slightly different from the water-filling result as there is no constant water level when delay cost is considered. This is because the allocation results $x_{i}(i=1,2, \ldots, I)$ is determined not only by the nonflexible load $l_{i}^{N}$, but also by another time interval dependent term $\frac{\delta}{2 \alpha}\left(i T \gamma^{-(I-i) T}\right)$. Indeed, solution (1) is of a water-filling like form if we interpret $\hat{w}_{i}=l_{i}^{N}+\frac{\delta}{2 \alpha}\left(i T \gamma^{-(I-i) T}\right)$ as the new modified nonflexible load in which $\frac{\delta}{2 \alpha}\left(i T \gamma^{-(I-i) T}\right)$ acts as an additional time related nonflexible load. The water level drops over time since later time intervals induce greater additional nonflexible load demands. It can be seen that the water level gets steeper as the delay cost weight $\delta$ increases. However, once the load demand of a time interval achieves $L_{M}$, no more load demands can be allocated to that interval. Thus after $\delta$ increases to a certain value, the optimal load profile becomes saturated (fixed). In this saturated profile, all except the last time interval with positive flexible load demands get $L_{M}$ amount of flexible load demands. The generation cost also achieves its maximum value corresponding to the saturated profile.

Figure 3 shows the saturated load profile (with $L_{M}$ normalized to 1 ) and generation costs (with the minimum normalized to 1 ) for different values of $\delta$, given the average initial load demands. Note that the generation cost is nondecreasing over $\delta$ 's, which is intuitively reasonable because the utility has smaller and smaller flexibility on the scheduling operation as $\delta$ increases.
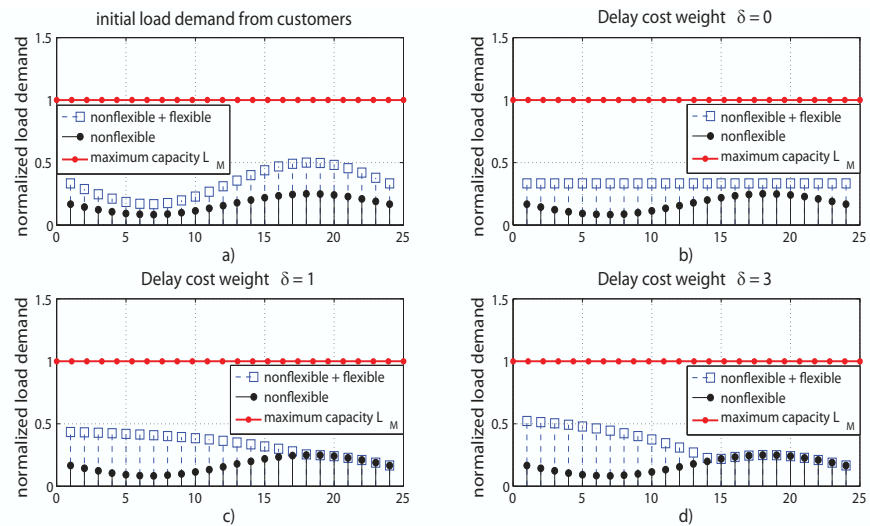

Fig. 2. Optimal load profile comparison for different delay cost weights $(\delta$ 's): a) Initial load demand from customers. b) Optimal load profile with no delay cost. c) Optimal load profile with delay cost weight $\delta=1$. d) Optimal load profile with delay cost weight $\delta=3$.
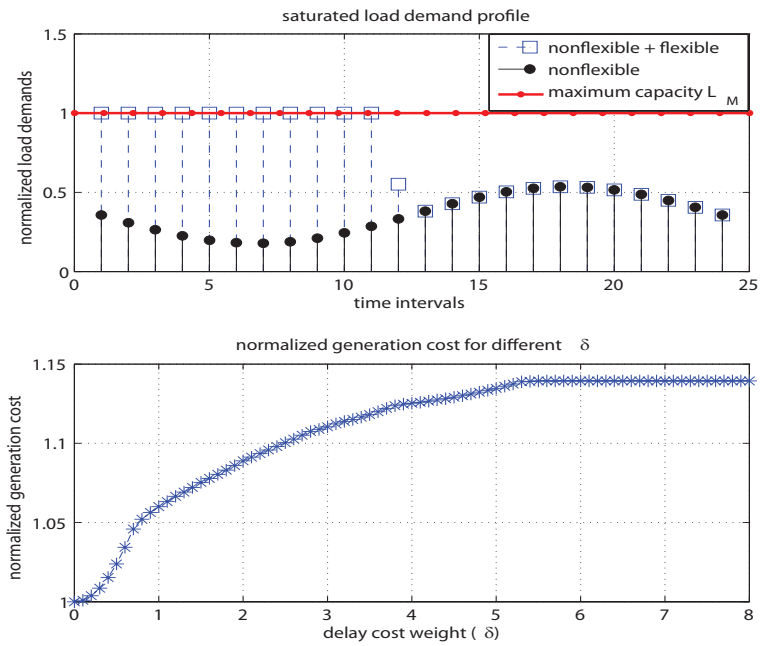

Fig. 3. Saturated optimal load demand profile (upper) and generation cost for different $\delta$ 's (lower)

Though the water-filling like solution is based on the specific forms of generation cost and delay cost, the decoupling framework still works for other cost functions. The resulting optimal allocation profile $x_{i}(i=1,2, \ldots, I)$ can always be used for next stage energy allocation among customers.

\section{Distributed Allocation among Customers: REPEATED VICKREY AUCTIONS}

Based on the optimal generation profile obtained in the first stage, a customer-wise allocation scheme is further needed for each time interval. The desired allocation scheme should have several properties: 1) Because the number of customers is large, as well as the status of customers (connected or disconnected) vary over time, the allocation scheme need to be robust to adding $\backslash$ removing customers. 2) Customers should be able to specify how much money they are willing to pay 
according to their own budgets. 3) Selfish customers, who are only interested in maximizing their own profit needs to be taken into account. Customers cheat on the amount of money they are willing to pay if doing so results in higher profit. Thus the desired scheme should be robust to customer cheating and should distribute electrical energy efficiently, meaning allocating energy to customers who really need it the most. Here we adopt repeated Vickrey auctions, which works well for all purposes above as will be shown.

Though the electrical energy is infinitely divisible, the concept of a basic unit, say $1 \mathrm{KWh}$, is needed to describe the minimum amount of energy to be functional for customers. Thus we assume that the utility is scheduling $\left\lceil x_{i}\right\rceil$ amount of load demand instead of $x_{i}(i=1,2, \ldots, I)$, where $\left\lceil x_{i}\right\rceil$ is the smallest integer no smaller than $x_{i}$. In the end of the block, all extra generated energy $\sum_{i=1}^{I}\left(\left\lceil x_{i}\right\rceil-x_{i}\right)$ will be stored. In the repeated auction scheme, for each time interval $i$ with positive $\left\lceil x_{i}\right\rceil$, all $K$ customers participate in a Vickrey auction for $\left\lceil x_{i}\right\rceil$ number of electrical energy units. No auction will be held in the rest time intervals. In the auction for time interval $i$, customer $k(k=1,2, \ldots, K)$ submits $\left\lceil x_{i}\right\rceil$ number of bids $b_{i, k}^{m}$ 's $\left(m=1,2, \ldots,\left\lceil x_{i}\right\rceil\right)$ to indicate how much it is willing to pay for each additional energy unit. Thus bid $b_{i, k}^{m}$ is the amount of money customer $k$ is willing to pay for its $m$-th energy unit. Let $\mathbf{b}_{i, k}=\left(b_{i, k}^{1}, b_{i, k}^{2}, \ldots, b_{i, k}^{\left\lceil x_{i}\right\rceil}\right)$ denote the bid vector of customer $k$ at time interval $i$. Assume $\mathbf{b}_{i, k}$ $\in B:=\left\{\mathbf{b}_{i, k} \in \mathbb{R}_{+}^{\left\lceil x_{i}\right\rceil} \mid b_{i, k}^{1} \geq b_{i, k}^{2} \geq \cdots \geq b_{i, k}^{\left\lceil x_{i}\right\rceil}, \forall k=\right.$ $1,2, \ldots, K\}$. Note that, in practice restricting bid vector to have non-increasing components makes sense. Because if a customer has no energy, it needs energy the most urgently and its valuation for its first unit of energy is the highest. As it gets more and more energy units, its demand gets saturated gradually and the marginal valuation is thus non-increasing. If a customer $k$ is only interested in getting $x_{i, k} \leq\left\lceil x_{i}\right\rceil$ number of energy units in the auction in time interval $i$, then the last $\left\lceil x_{i}\right\rceil-x_{i, k}$ elements of its bid vector are all zeros. In the auction for time interval $i$, a total of $K \times\left\lceil x_{i}\right\rceil$ bids $b_{k}^{m}$ 's $\left(k=1,2, \ldots, K ; m=1,2, \ldots,\left\lceil x_{i}\right\rceil\right)$ are placed and the $\left\lceil x_{i}\right\rceil$ energy units are awarded to the $\left\lceil x_{i}\right\rceil$ highest of these bids, which are deemed winning bids. Ties are broken by choosing with equal probability among all possible rankings among tying bids. The number of energy units awarded to a customer is equal to the number of winning bids submitted by that customer. Thus if customer $k$ has $m_{i, k} \leq\left\lceil x_{i}\right\rceil$ of the highest bids, then it is awarded $m_{i, k}$ units of electrical energy at time interval $i$. Denote by $\mathbf{c}^{-k}$ the $\left\lceil x_{i}\right\rceil$-vector of competing bids facing customer $k$, so that $c_{1}^{-k}$ is the highest of the other bids, $c_{2}^{-k}$ is the second highest of the other bids, and so on. To win exactly $m$ energy unit, customer $k$ 's $m$-th highest bid must defeat the $m$-th lowest competing bid. If customer $k$ wins $m_{i, k}$ units of electrical energy, then the amount it pays is the sum of $m_{i, k}$ highest losing bids of the other customers, which is $\sum_{m=1}^{m_{i, k}} c_{\left\lceil x_{i}\right\rceil-m_{k}+m}^{-k}$.

\section{A. Truthful Bidding Strategy for One-shot Vickrey Auction}

In the auction of each time interval, all customers have their own valuations, which determine the bidding strategies, attached to all energy units for sale. In the electricity market, customers do not know other's valuations precisely (incomplete information) since valuations of different customers are determined by their own energy needs and consumption behaviors (private valuation). Denoted by $\mathbf{v}_{i, k}=\left[v_{i, k}^{1}, v_{i, k}^{2}, \ldots, v_{i, k}^{\left\lceil x_{i}\right\rceil}\right]$ the private valuation vector of customer $k$ at time interval $i$, where $v_{i, k}^{m}$ represents the marginal value of obtaining the $m$-th unit of energy. These marginal values are assumed to be non-increasing so that $v_{i, k}^{1} \geq v_{i, k}^{2} \geq \cdots \geq v_{i, k}^{\left\lceil x_{i}\right\rceil}, \forall k$, for similar reasons when we assumed non-increasing marginal bids. The total value to the customer $k$ of obtaining exactly $m_{i, k} \leq\left\lceil x_{i}\right\rceil$ units is then the sum of the first $m_{i, k}$ marginal values: $\sum_{j=1}^{m_{i, k}} v_{i, k}^{j}$. Note that symmetry on valuations is usually assumed in Vickrey auction literature ( [11], [12]), in which $\mathbf{v}_{i, k}$ 's are independently and identically distributed (i.i.d) on the valuation set $\mathbf{V}_{i, k}=\left\{\mathbf{v}_{i, k} \in\left[0, \omega_{i}\right]^{\left\lceil x_{i}\right\rceil}: \forall m, v_{i, k}^{m} \geq v_{i, k}^{m+1}\right\}$, where $\omega_{i}$ is the maximum valuation for all customers. However, the i.i.d symmetric condition might be too strong for our problem since the valuations of different customers could be different depending on individual consumption behaviors. Thus we drop the condition of identical distribution and assume more general asymmetric customers - customer $k$ 's valuation vector $\mathbf{v}_{i, k}$ is independently drawn from some distribution $F_{i, k}$ that has positive density everywhere on the set $\mathbf{V}_{i, k}$.

The Vickrey auction in each time interval actually forms a game with incomplete information, in which every customer wants to maximize its own payoff. Here a customer's payoff equals the sum of valuations obtained from winning energy units minus the total payment. Under the assumptions above, it can be proved that the Vickrey auction is incentive compatible, meaning truthful bidding (bidding the real value) maximizes each customer's payoff [11]. Thus truthful bidding is a weakly dominant strategy for every customer and thus forms a Bayesian Nash equilibrium.

\section{B. Bayesian Nash Equilibria Structure: General Analysis}

Though every customer's payoff is maximized in the truthful bidding equilibrium, Vickrey auction does not guarantee the benefit of the utility. This is because truthful bidding is only a weakly dominant strategy and truthful bidding equilibrium is not the unique Bayesian Nash equilibrium in a one shot Vickrey auction. Therefore detailed analysis on the entire equilibrium solution set of Vickrey auction is necessary for finding better allocation schemes in our second stage scheduling. Based on [13], we divide the Bayesian Nash equilibria into two categories.

Equilibria in the first category can be described as follows: There exists at least one customer $k$ who has at least one bid $b_{i, k}^{m} \in\left(0, \omega_{i}\right)$ with positive probability. There is a threshold $b_{i}^{*} \in\left(0, \omega_{i}\right)$ for all customers such that all customers bid truthfully for which they have a valuation exceeding $b_{i}^{*}$. 
Furthermore, there is a unique distinct customer $\hat{k}$ who bids $b_{i}^{*}$ on any unit for which his valuation is below the threshold. The remaining customers bid zero on any unit for which their valuation is below the threshold. Put in a more mathematical format:

$$
b_{i, \hat{k}}^{m}=\left\{\begin{aligned}
v_{i, \hat{k}}^{m} & \text { if } v_{i, \hat{k}}^{m} \in\left[b_{i}^{*}, \omega_{i}\right] \\
b_{i}^{*} & \text { if } v_{i, \hat{k}}^{m} \in\left[0, b_{i}^{*}\right)
\end{aligned}\right.
$$

for all $m=1,2, \ldots,\left\lceil x_{i}\right\rceil$ and

$$
b_{i, k}^{m}=\left\{\begin{aligned}
v_{i, k}^{m} & \text { if } v_{i, k}^{m} \in\left(b_{i}^{*}, \omega_{i}\right] \\
0 & \text { if } v_{i, k}^{m} \in\left[0, b_{i}^{*}\right]
\end{aligned}\right.
$$

for all $k \neq \hat{k}$ and all $m=1,2, \ldots,\left\lceil x_{i}\right\rceil$ and $b_{i}^{*}:=\inf \{b \in$ $\left(0, \omega_{i}\right) \mid \exists k, m$ s. t. $\left.\forall \epsilon>0, \operatorname{Pr}\left\{b_{i, k}^{m} \in[b, b+\epsilon]\right\}>0\right\}$. If we make stronger assumptions on the customers' valuation distribution, say assuming there exists at least one customer whose valuation distribution over $\left(0, \omega_{i}\right)$ assigns positive probability to arbitrarily small positive values, which is reasonable since in reality customers usually have continuous distribution over the valuation set. Then we have $b_{i}^{*}=0$ and the first category equilibria reduce to the truthful bidding equilibrium.

For all equilibria that are not in the first category, there is zero probability of positive bids below the highest valuation $\omega_{i}$. Each customer $k(k=1,2, \ldots, K)$ bids at or above the highest valuation $\omega_{i}$ on $\hat{m}_{i, k}$ number of units and bids zero on the remaining units in such a manner that the total number of positive bids across all customers equals the number of units to be sold, i.e. $\sum_{k=1}^{K} \hat{m}_{i, k}=\left\lceil x_{i}\right\rceil$. The second category of Bayesian Nash equilibria reveals the possibility that the Vickrey auction might end up with a collusive equilibrium that customers bid untruthfully and all energy units are sold at a price of zero, resulting negative profit of the utility.

Generally speaking, equilibria of both categories are collusive in the sense that there are positive probabilities that customers get some energy units at a price of zero. To guarantee the minimum profit covers the generation cost, it is reasonable for the utility to set a positive reserve price $r_{i}$ for the auction in time interval $i$ such that each customer has to pay at least the reserve price for any unit obtained. It has been proved in [13] that with a positive reserve price $r_{i}$, the auction with more than two customers converges to a unique Bayesian Nash equilibrium, in which customers refrain from bidding on any unit for which their valuation is less than $r_{i}$ and otherwise bid their valuation for each unit. Thus the Vickrey auction with a reserve price gives a better allocation scheme in the sense that 1) It is robust to collusion by customers, 2) It guarantees the minimum profit of the utility and 3) It converges to the unique Bayesian Nash equilibrium.

\section{Valuation and Bidding Strategy in Repeated Auctions}

In the second stage, energy allocation among customers is conducted one time interval after another. We assume the energy units are not time interval specific, meaning energy provided in different time intervals are equally functional to customers. Then valuations on energy units provided in earlier time intervals will be lower than those provided in later time intervals. This is because the more time intervals left, the more chances for a customer to win energy units for its load demands. We thus model customers' valuations over time intervals as a product of the basic valuations and a time scaling factor. Considering that the valuations in the last round Vickrey auction is exactly the same as the valuations in a one-shot Vickrey auction, we denote by $v_{k}^{m}$ the valuation of customer $k$ for its $m$-th energy unit in a one-shot Vickrey auction, its valuation in time interval $i$ is given by $v_{k, i}^{m}=v_{k}^{m} \eta^{-(I-i) T}$ where $\eta$ is the time scaling factor. Note that for a certain customer, if it has already won $u$ units of energy in previous auctions, then its highest valuation in current auction is for its $(u+1)$-th energy unit assuming the non-increasing marginal valuations.

Different from valuations, in repeated auctions determining the bidding strategies of customers in general is a difficult problem in auction theory literature [11]. In our problem, the individual bidding strategy can be affected by too many factors. For example, a customer who is active in current auction and fails to win will bid higher in the next auction. This is due to the deterioration of available energy units relative to current load demands. However on the other hand, the higher bids from those who did not win in the previous auction seems to be mitigated by the fact that there might be fewer competing bids in the current auction. This is because customers whose load demands are fully supported will quit from the following auctions automatically and the currently active customers who won some energy units in previous auctions will have lower (at most equal) valuations on their additional energy units. Thus a less fierce competition could be expected. As can be seen, the two factors mentioned above both exist in our problem but lead to opposite bidding behaviors in repeated auctions. Another major difficulty comes from the fact that in repeated Vickrey auctions, once a bidder wins some units, its behavior and interest are different from others. In other words, even if customers are symmetric ex ante, multi-unit demands introduce asymmetries among customers in later auctions, which deviates the basic requirement for analyzing symmetric bidding strategy Bayesian Nash equilibria [11].

In auction theory, a bidding strategy or bidding function is a mapping from valuation set to bid set. An auction is called a standard auction if items for sale are allocated to highest bids. An auction is efficient (maximizing bidder social welfare) if items for sale are allocated to highest valuations. It has been proved that an equilibrium of a standard auction is efficient if and only if the bidding function is a single increasing function [11]. It is easy to see the Vickrey auction is a standard auction. The bidding function in the truthful bidding 
equilibrium is actually the identity function which leads to an efficient mechanism under the non-increasing valuation assumed above.

\section{Simulation Result}

In this section we implement a 24 hour block scheduling for an electricity market, which consists of 1 utility and 5000 residential households with each household of 4 people on average. According to the data in [14] and Electrical Information, from 2006 to 2008 the average electricity price in U.S.A. is 9.28 cents per $\mathrm{KWh}$ and the average energy consumption of one person is about $39.39 \mathrm{KWh}$ per day. Thus it is reasonable to assume that the load demands for a household in different time intervals are uniformly distributed between 0 and some maximum value. The maximum value is time dependent and ranges from 10 to $20 \mathrm{KWh}$. Moreover we assume that customers' valuations are uniformly distributed over $(6,10)$ cents. Fig. 4 shows utility's average profits (payments from customers minus the total generation cost) for different reserve prices and different time interval sizes in repeated Vickrey auctions. Note that utility's profit increases first as the reserve price increases from below to above the generation cost, after a certain point the profit decreases because the amount of energy sold decreases. Utility's profit drops to zero when the reserve price is $0.1 \$$ since all valuations from customers are below the reserve price and no energy is sold. It can be seen that greater time interval size gives greater utility's profit for the optimal reserve price, but more severe degradation for nonoptimal reserve prices.

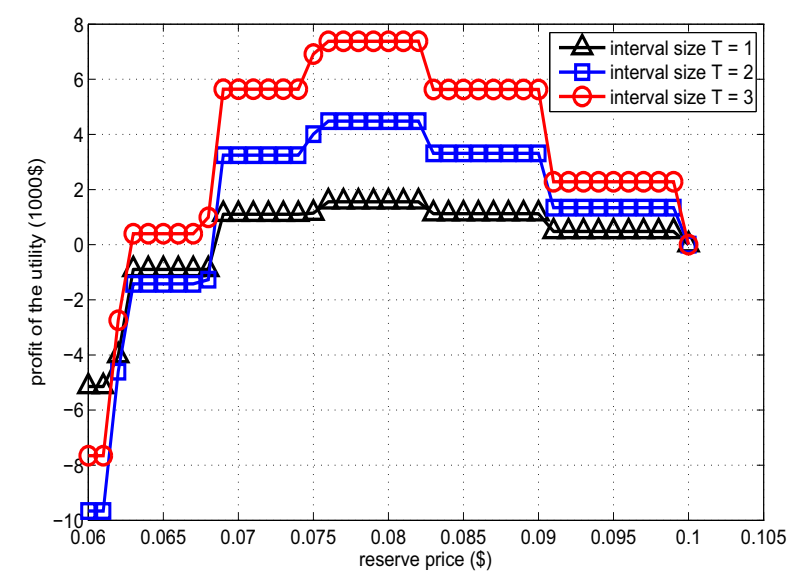

Fig. 4. Utility's profit for different reserve prices and different time interval sizes

\section{CONCLUSION}

In this paper, a block processing model with finite time intervals was adopted for DR $\backslash$ DSM scheduling. The two principal objectives: utility cost minimization and customer social welfare maximization were decoupled into two stages. In the first stage, minimizing the weighted sum of generation cost and delay operation cost of utility was formulated as a convex optimization problem, and the optimal time interval reallocation solution was derived. It was shown that the average generation cost increases as the delay cost weight increases until the optimal load demand profile saturates. Based on this optimal load profile derived in the first stage, repeated Vickrey auctions over time intervals were adopted to allocate load demands among customers. We showed that truthful bidding is a weakly dominant strategy for all customers in a one shot Vickrey auction. We also showed that collusive equilibria do exist and can severely jeopardize utility's profit. We then generalized our discussion to the structure of the entire set of Bayesian Nash equilibria of one shot Vickrey auction, which can be divided into two categories. Since both categories of equilibria are collusive in general, we further showed that if the utility introduce a positive reserve price, the Vickrey auction becomes more robust to collusion by customers and the resulting unique Bayesian Nash equilibrium guarantees the basic profit of the utility. Assuming increasing valuations over time intervals, we simulated our block processing based model of a electricity market in which the influences of reserve price and time interval size on utility's profit was emphasized.

\section{REFERENCES}

[1] “The Smart Grid: An Introduction,” Tech. Rep., The U.S. Department of Energy, Oct. 2008.

[2] "Benefits of demand response in electricity markets and recommendations for achieving them," Tech. Rep., U.S. Department of Energy, Feb. 2006.

[3] V. Bakker, M. Bosman, A. Molderink, J. Hurink, and G. Smit, "Demand side load management using a three step optimization methodology," in 2010 First IEEE International Conference on Smart Grid Communications (SmartGridComm), (Gaithersburg, MD, USA), pp. 431-436, Oct. 2010.

[4] S. Hatami and M. Pedram, "Minimizing the electricity bill of cooperative users under a quasi-dynamic pricing model," in 2010 First IEEE International Conference on Smart Grid Communications (SmartGridComm), (Gaithersburg, MD, USA), pp. 421-426, Oct. 2010.

[5] P. Samadi, A. Mohsenian-Rad, R. Schober, V. Wong, and J. Jatskevich, "Optimal real-time pricing algorithm based on utility maximization for smart grid," in 2010 First IEEE International Conference on Smart Grid Communications (SmartGridComm), (Gaithersburg, MD, USA), pp. 415 - 420, Oct. 2010.

[6] S. Kishore and L. Snyder, "Control mechanisms for residential electricity demand in smartgrids," in 2010 First IEEE International Conference on Smart Grid Communications (SmartGridComm), (Gaithersburg, MD, USA), pp. 443-448, Oct. 2010.

[7] S. Caron and G. Kesidis, "Incentive-based energy consumption scheduling algorithms for the smart grid," in 2010 First IEEE International Conference on Smart Grid Communications (SmartGridComm), (Gaithersburg, MD, USA), pp. 391-396, Oct. 2010.

[8] C. Ibars, M. Navarro, and L. Giupponi, "Distributed demand management in smart grid with a congestion game," in 2010 First IEEE International Conference on Smart Grid Communications (SmartGridComm), (Gaithersburg, MD, USA), pp. 495-500, Oct. 2010.

[9] L. Chen, N. Li, S. Low, and J. Doyle, "Two market models for demand response in power networks," in 2010 First IEEE International Conference on Smart Grid Communications (SmartGridComm), (Gaithersburg, MD, USA), pp. 397-402, Oct. 2010.

[10] D. Li, S. K. Jayaweera, O. Lavrova, and R. Jordan, "Load management for price-based demand response scheduling $\mathrm{f}$ a block scheduling model," in International Conference on Renewable Energies and Power Quality (ICREPQ'11), (Las Palmas de Gran Canaria, Spain), Apr. 2011.

[11] V. Krishna, Auction Theory. Academic Press, Elsevier, 2010.

[12] D. Easley and J. Kleinberg, Networks, Crowds, and Markets: Reasoning about a Highly Connected World. Cambridge University Press, 2010.

[13] A. Blume, P. Heidhues, J. Lafky, J. Mnster, and M. Zhang, "All equilibria of the multi-unit vickrey auction," Games and Economic Behavior, vol. 66, no. 2, pp. 729-741, 2009.

[14] R. Soultanian, "Global electricity pricing: Ups and downs of global electricity prices," Power Engineering International Magazine, Jul. 2007. 